01

\title{
Присоединенная волна круглого открытого диэлектрического волновода
}

\author{
(C) В.А. Малахов, А.С. Нечаев, А.С. Раевский, С.Б. Раевский \\ Нижегородский государственный технический университет им. Р.Е. Алексеева, Нижний Новгород, Россия \\ E-mail: physics@nntu.ru
}

Поступило в Редакцию 9 апреля 2019г.

В окончательной редакции 30 апреля 2019 г.

Принято к публикации 31 мая 2019 г.

\begin{abstract}
Показана возможность существования в круглом открытом диэлектрическом волноводе, находящемся в неограниченной однородной среде, присоединенных волн, которые описываются решениями однородной краевой задачи.
\end{abstract}

Ключевые слова: присоединенные волны, диэлектрический волновод, краевая задача, комплексные волны.

DOI: 10.21883/PJTF.2019.17.48222.17823

Исследование спектров волн направляющих структур сводится к решению краевых задач на однородном уравнении Гельмгольца относительно продольных компонент векторов Герца. Данные краевые задачи для цилиндрических волноводов относятся в общем случае к классу несамосопряженных [1-4], их собственные значения в общем случае являются комплексными величинами [2].

Кроме того, для направляющих структур могут быть сформулированы присоединенные краевые задачи, состоящие из дифференциальных уравнений и систем граничных условий, которые образуются [2] дифференцированием по собственному значению исходных дифференциального уравнения и граничных условий соответственно. Решения присоединенных краевых задач соответствуют так называемым присоединенным волнам [3], характерной особенностью которых является наличие линейной зависимости их амплитуд от продольной координаты.

В работах [1,4] были исследованы дисперсионные характеристики волн круглых многослойных экранированных волноводов, приведены решения присоединенной краевой задачи, сделан вывод о возможности существования присоединенной волны в точках жордановой кратности волновых чисел [5]. В настоящей работе показано существование присоединенных волн в круглом открытом диэлектрическом волноводе (ДВ) в неограниченной однородной среде (рис. 1), описываемых решениями краевой задачи на однородном уравнении Гельмгольца. Волновод представляет собой диэлектрический стержень радиуса $a$ с относительной диэлектрической проницаемостью $\tilde{\varepsilon}_{1}$ (область 1 ), помещенный в среду с относительной диэлектрической проницаемостью $\tilde{\varepsilon}_{2}$ (область 2).

Поля волн рассматриваемой структуры выражаются через продольные компоненты векторов Герца $\Pi_{z}^{e, m}$, которые удовлетворяют уравнению Гельмгольца

$\frac{\partial^{2} \Pi_{z}^{e, m}}{\partial r^{2}}+\frac{1}{r} \frac{\partial \Pi_{z}^{e, m}}{\partial r}+\frac{1}{r^{2}} \frac{\partial^{2} \Pi_{z}^{e, m}}{\partial \phi^{2}}+\frac{\partial^{2} \Pi_{z}^{e, m}}{\partial z^{2}}+\varepsilon \mu \omega^{2} \Pi_{z}^{e, m}=0$,

где $r, \phi, z$ - цилиндрические координаты, и условию ограниченности в пределах поперечного сечения ДВ.
Граничное условие при $r \rightarrow \infty$ не накладывается в предположении, что в направляющей структуре могут распространяться как собственные, так и несобственные волны.

Тангенциальные компоненты полей, выраженные через электрический и магнитный векторы Герца $\Pi_{z}^{e, m}$, должны удовлетворять граничным условиям

$$
\begin{array}{ll}
E_{z 1}(r=a)=E_{z 2}(r=a), & H_{z 1}(r=a)=H_{z 2}(r=a), \\
E_{\phi 1}(r=a)=E_{\phi 2}(r=a), & H_{\phi 1}(r=a)=H_{\phi 2}(r=a),
\end{array}
$$

Решение уравнения (1) может быть представлено в виде $[4,5]$ :

$$
\Pi_{z}^{e, m}=[R(r) f(z)+\bar{R}(r) \bar{f}(z)]\left\{\begin{array}{l}
\cos n \phi, \\
\sin n \phi,
\end{array}\right.
$$

где функции $R(r), f(z), \bar{R}(r), \bar{f}(z)$ удовлетворяют уравнениям

$$
\begin{gathered}
R^{\prime \prime}(r)+R^{\prime}(r) / r+\left(\alpha^{2}-n^{2} / r^{2}\right) R(r)=0, \\
\bar{R}^{\prime \prime}(r)+\bar{R}^{\prime}(r) / r+\left(\alpha^{2}-n^{2} / r^{2}\right) \bar{R}(r)=R(r), \\
\bar{f}^{\prime \prime}(z)+\beta^{2} \bar{f}(z)=0, \\
f^{\prime \prime}(z)+\beta^{2} f(z)=-\bar{f}(z) .
\end{gathered}
$$

Волновые числа $\alpha$ и $\beta$ (поперечное и продольное) в областях 1 и 2 связаны соотношениями

$$
\varepsilon_{1,2} \mu_{1,2} \omega^{2}=\alpha_{1,2}^{2}+\beta^{2} .
$$

Уравнения (5) и (7) являются [4] присоединенными к уравнениям (4) и (6).

Векторы Герца после подстановки в них решений уравнений (4)-(7) запишутся как

$$
\begin{aligned}
\Pi_{z q}^{e, m}= & {\left[C_{n q}^{e, m} R_{n q}^{e, m}\left(\alpha_{q} r\right)+D_{n q}^{e, m}\left(-\frac{i z}{2 \beta}\right) R_{n q}^{e, m}\left(\alpha_{q} r\right)\right.} \\
& \left.+D_{n q}^{e, m} \rho_{n q}\left(\alpha_{q} r\right)\right]\left\{\begin{array}{c}
\cos n \phi \\
\sin n \phi
\end{array}\right\} e^{-i \beta z},
\end{aligned}
$$


где $q$ - номер области, $R_{n q}^{e, m}\left(\alpha_{q} r\right)-$ функция Бесселя (во внутренней области) или функция Ханкеля второго рода (во внешней области), $\rho_{n q}\left(\alpha_{q} r\right)-$ решение присоединенного уравнения Бесселя

$$
\begin{gathered}
\rho^{\prime \prime}\left(\alpha_{1,2} r\right)+\frac{1}{r} \rho^{\prime}\left(\alpha_{1,2} r\right)+\left(\alpha_{1,2}^{2}-\frac{n^{2}}{r^{2}}\right) \rho\left(\alpha_{1,2} r\right) \\
=\bar{D}_{n_{1,2}}^{e, m} R_{n}^{e, m}\left(\alpha_{1,2} r\right),
\end{gathered}
$$

в котором $\bar{D}_{n_{1,2}}^{e, m}-$ амплитудные коэффициенты соответствующих векторов Герца (постоянные интегрирования уравнения Бесселя во внутренней и внешней областях ДВ).

При выполнении условия $\bar{D}_{n_{1,2}}^{e, m}=D_{n_{1,2}}^{e, m}$ решение (8) удовлетворяет однородному уравнению Гельмгольца. При невыполнении этого условия функция (8) является решением присоединенного уравнения Гельмгольца, у которого в правой части стоит решение соответствующей однородной краевой задачи.

Компоненты полей, выраженные через электрический и магнитный векторы Герца (8), подставляются в граничные условия (2). Приравнивая в граничных условиях члены, пропорциональные $z$, получаем систему четырех линейных однородных алгебраических уравнений относительно коэффициентов $D_{n_{1,2}}^{e, m}$ :

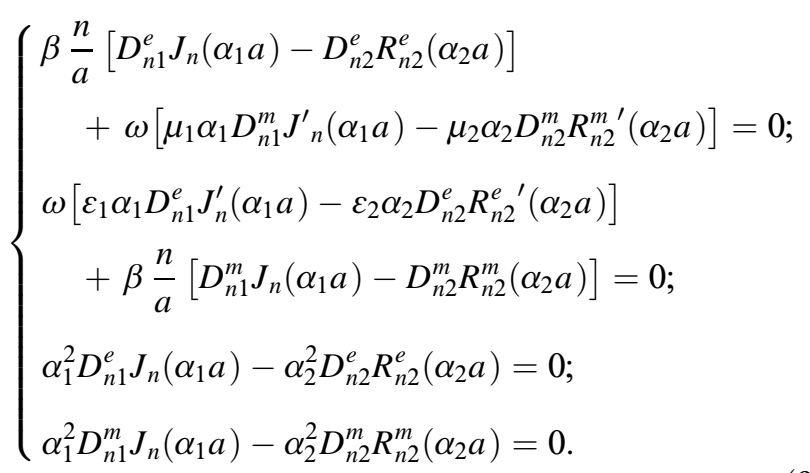

Приравнивая в граничных условиях члены, не зависящие от $z$, получаем систему четырех линейных неоднородных алгебраических уравнений относительно $C_{n 1,2}^{e, m}$

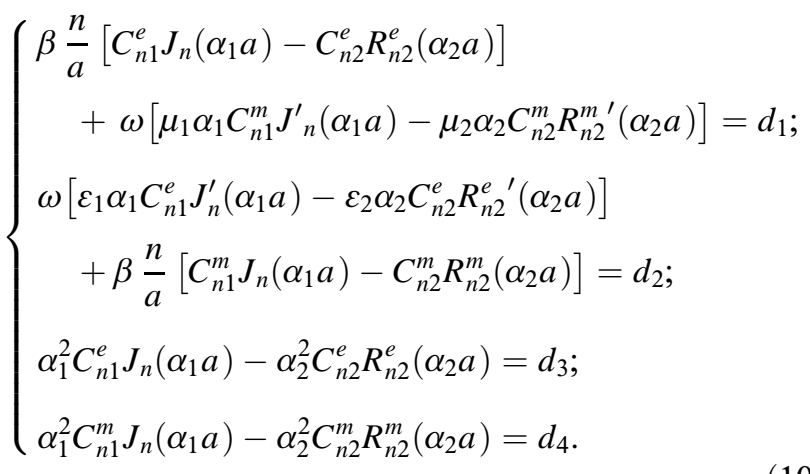

Главные определители систем уравнений (9), (10) совпадают и оказываются тождественными определителю системы линейных однородных алгебраических

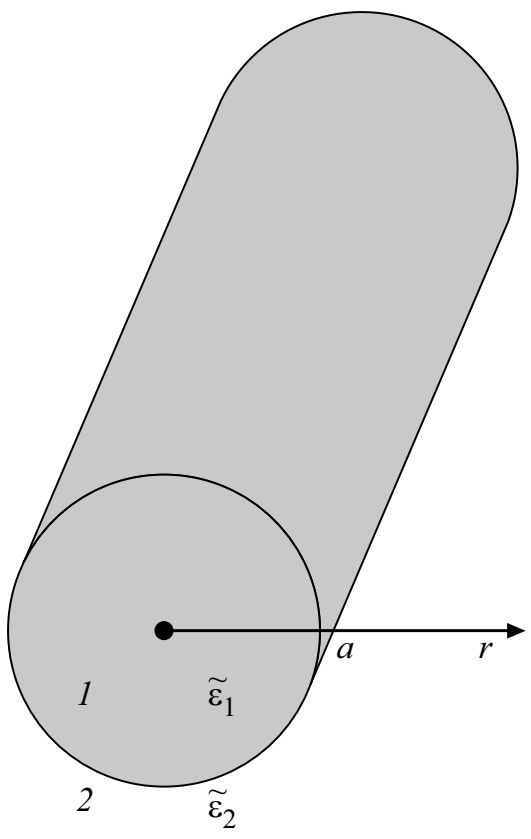

Рис. 1. Круглый открытый диэлектрический волновод.

уравнений, получаемых из граничных условий (2) в случае нормальных волн круглого открытого ДВ. Решения систем линейных однородных и неоднородных уравнений с одинаковыми главными определителями могут совпадать только при равенстве нулю дополнительных определителей систем неоднородных линейных алгебраических уравнений.

Выражение коэффициентов $D_{n 2}^{e, m}$ через $D_{n 1}^{e, m}$ и $C_{n 2}^{e, m}$ через $C_{n 1}^{e, m}$ приводит к понижению вдвое порядка систем уравнений. Из условия нетривиальности решения редуцированной системы линейных однородных уравнений второго порядка получаем дисперсионное уравнение волн круглого открытого ДВ

$$
\left|\begin{array}{ll}
a_{11} & a_{12} \\
a_{21} & a_{22}
\end{array}\right|=0 .
$$

Редуцированная система линейных неоднородных алгебраических уравнений при равенстве нулю ее дополнительных определителей

$$
\begin{aligned}
& \left|\begin{array}{ll}
c_{1} & a_{12} \\
c_{2} & a_{22}
\end{array}\right|=0, \\
& \left|\begin{array}{ll}
a_{11} & c_{1} \\
a_{21} & c_{2}
\end{array}\right|=0,
\end{aligned}
$$

где $c_{1}$ и $c_{2}-$ правые части редуцированной системы линейных неоднородных алгебраических уравнений, может иметь решения, общие с системой линейных однородных алгебраических уравнений.

Совокупность трех трансцендентных уравнений (11), (12a) и (12b) образует дисперсионную задачу для при- 


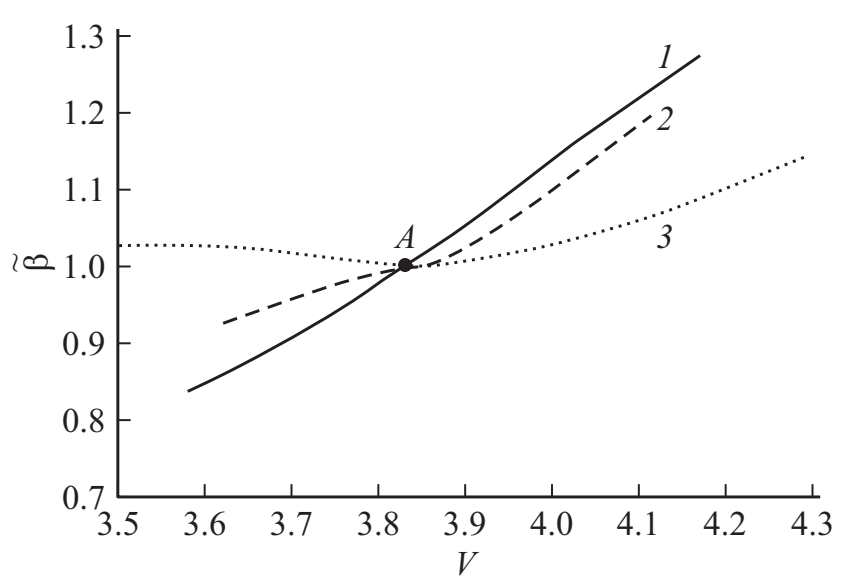

Рис. 2. Решения уравнений, образующих дисперсионную задачу для присоединенных волн круглого открытого диэлектрического волновода с параметрами $\tilde{\varepsilon}_{1}=9, \quad \tilde{\varepsilon}_{2}=1$.
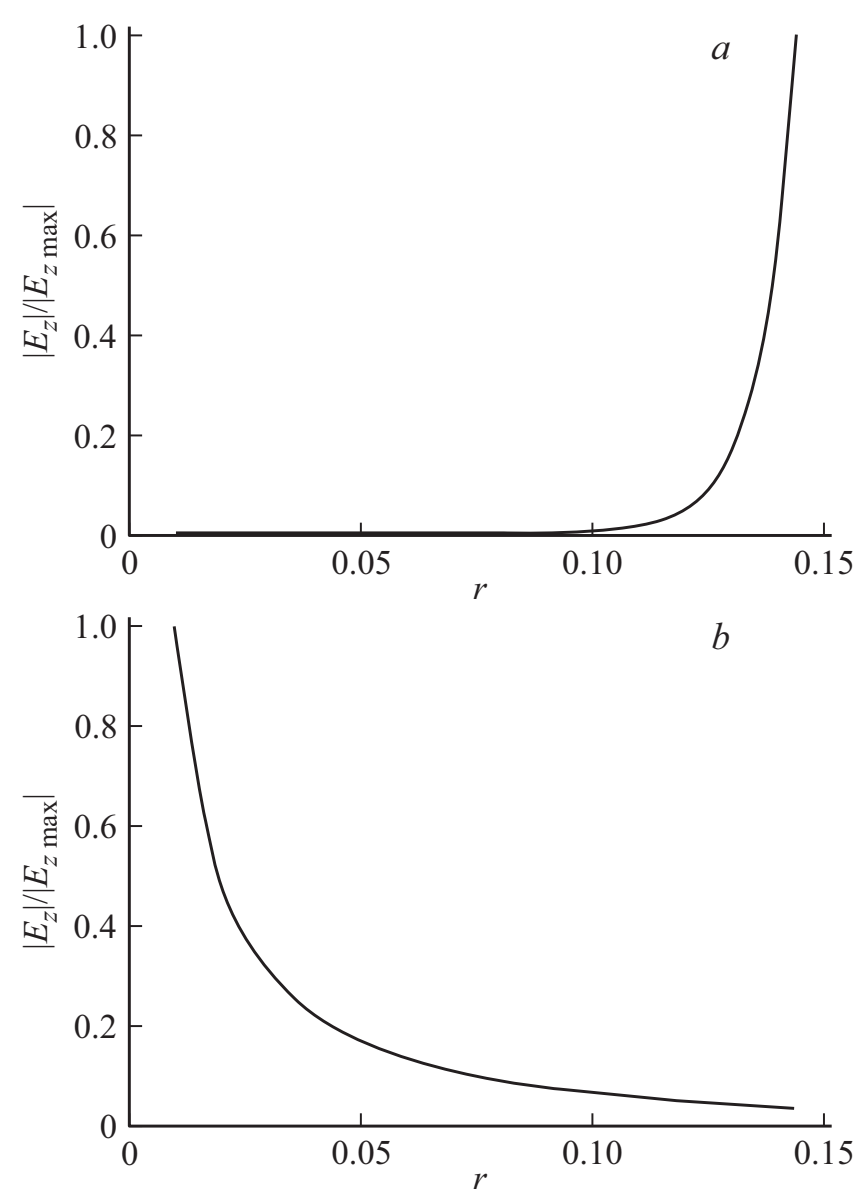

Рис. 3. Распределение нормированного модуля компоненты электрического поля $\left|E_{z}\right| / \mid E_{z}$ max $\mid$ вдоль радиальной координаты вне диэлектрического стержня. $a-$ на нормированной частоте $V=3.85, b-$ на нормированной частоте $V=3.9$.

соединенных волн круглого открытого ДВ. Для нахождения совместных решений уравнений (11), (12a) и (12b) был использован комбинированный численный метод поиска комплексных корней трансцендентных уравнений [6], объединяющий в себе метод Мюллера [7] и метод вариации фазы [1]. Этот метод сочетает быстроту нахождения комплексных корней дисперсионного уравнения и надежность идентификации корня. При этом исключаются ложные корни (локальные экстремумы), возможные при использовании метода Мюллера.

На рис. 2 приведены решения уравнения (11) (линия 1 , соответствующая дисперсионной характеристике волны $E H_{11}$ ), уравнения (12a) (линия 2) и уравнения (12b) (линия 3). Точка $A-$ общее решение уравнений (11), (12a) и (12b). На рис. $2 V=k_{0} a \sqrt{\tilde{\varepsilon}_{1}-\tilde{\varepsilon}_{2}}-$ нормированная частота, где $k_{0}-$ волновое число плоской волны в свободном пространстве, $a$ - радиус ДВ, $\tilde{\varepsilon}_{1}=9, \tilde{\varepsilon}_{2}=1 ; \tilde{\beta}=\beta / k_{0}-$ действительная часть нормированного продольного волнового числа.

Численные исследования показали, что общее решение уравнений (11), (12a), (12b) (точка $A$ на рис. 2) находится на нормированной частоте $V \approx 3.87$.

Для исследования особенностей поля, соответствующего полученному решению, был проведен расчет распределений нормированного модуля компоненты поля $\left|E_{z}\right| /\left|E_{z \max }\right|$ волны $E H_{11}$ вдоль радиальной координаты вне стержня (рис. $3, a, b$ ). Из рис. $3, a$ следует, что при переходе к нормированной частоте $V=3.85$ напряженность поля становится возрастающей по радиальной координате. Результаты решения дисперсионной задачи показали, что значение поперечного волнового числа в области 2 ДВ $\alpha_{2}$ находится в первом квадранте комплексной плоскости $\alpha_{2}$. Следовательно, можно утверждать, что полученные решения соответствуют вытекающей волне. Поскольку вытекающая волна является несобственной, решения дисперсионного уравнения, ей соответствующие, лишь условно можно называть собственными значениями. Более корректно их называть характеристическими числами.

Результаты решения дисперсионной задачи и расчеты распределений поля волны $E H_{11}$ на частотах $V>3.87$ показали, что волна $E H_{11}$ в данной частотной области является поверхностной: на нормированной частоте $V=3.9$ напряженность поля экспоненциально убывает с удалением от волновода.

Таким образом, можно сделать вывод, что точка $A$ (рис. 2), соответствующая решению дисперсионной задачи для присоединенных волн, является точкой перехода поверхностной волны $E H_{11}$ в вытекающую волну. В указанной точке найденная присоединенная волна обеспечивает полноту системы волн круглого открытого ДВ и обязательно должна учитываться при постановке дифракционных задач.

\section{Финансирование работы}

Представленные в статье исследования выполнены при финансовой поддержке Российского научного фонда, грант № 17-19-01628. 


\section{Конфликт интересов}

Авторы заявляют, что у них нет конфликта интересов.

\section{Список литературы}

[1] Раевский А.С., Раевский С.Б. Комплексные волны. М.: Радиотехника, 2010. 223 с

[2] Наймарк М.A. Линейные дифференциальные операторы. М.: Наука, 1969. 526 с.

[3] Ильинский А.С., Слепян Г.Я. Колебания и волны в электродинамических системах с потерями. М.: Изд-во МГУ, 1983. $231 \mathrm{c}$.

[4] Малахов В.А., Раевский А.С., Раевский С.Б. // Письма в ЖТФ. 2011. Т. 37. В. 2. С. 71-79.

[5] Краснушкин П.Е., Федоров Е.Н. // Радиотехника и электроника. 1972. Т. 17. № 6. С. 1129-1140.

[6] Малахов В.А., Раевский А.С., Раевский С.Б. // Журн. вычисл. математики и мат. физики. 2015. Т. 55. № 6. C. $1028-1038$. DOI: $10.7868 / \mathrm{S} 0044466915060095$

[7] Muller D.E. // Math. Comp. 1956. V. 10. P. 208-215. DOI: $10.1090 / \mathrm{S} 0025-5718-1956-0083822-0$ 